Page numbers in italics refer to illustrations and diagrams.

access ways $13,14,15$

see also ramps

dynamic capacity $27-28$

aesthetics of design 200, 201-2, 202

circular sloping deck types 124,125

air change rates, ventilation 192

aisles

angled stalls 11

dead ends 38

inflow capacity 36

minimum widths

one-way-flows 13

two-way-flows 13

pedestrians in 11

with angled parking stalls 11

ramp entries 21

ramp projections into 17

reduced

one-way-flows 11,19

two-way-flows 12

turning between 12,13

vehicles crossing 26-7

viewing angles 8

widths $10-11$

angled stalls 10

angles 11,12

circulation efficiency 38-40, 39

design implications 39-40, 39

dynamic efficiency 29

minimum dimensions 13

pedestrians in aisles 11

ramp widths 21

\section{barriers 186}

exit 38

numbers of 189

two-way-flow 188

Birmingham airport, car park 152

camper vans 6

capacities

medium stay car parks 31

short stay car parks 31

tidal car parks 32

car parks

see also multi-storey car parks

as motorists' destination influences 1-2

user friendly 2, 37-9

CCTV 168, 169-70

optimum monitoring 169-70

presence of 169

changes of use, car parks 2-3

circular decks, two-way-flow 126, 127

circular ramps 22, 24

end

one-way-flow 114,115

two-way-flow 116, 117 full, two-way-flow 140, 141

turning circles 139

two full, one-way-flow 142, 143

circular sloping decks 124,125

see also CSD series

user-unfriendliness 125

circulation design, simplicity $37-8$

circulation efficiency

angled stalls $38-40,39$

crossovers 38

importance of 35-6

indicating 34

combined flat and sloping decks

internal cross-ramps

see also VCM and WPD series

pedestrian access 91

combined helix

one- and two-way-flows

side connected $70,71,80,81$

one-way-flow, side connected 82,83

contra-flow rapid exit, one-way-flow types 56,57

control signs 178

control systems

barriers 186

exit 38

numbers of 189

two-way-flow 188

disabled drivers 163

pay and display 187

payment by mobile phone $187-8$

payment on exit 186,187

payment on foot 187

tag systems 188

crossovers $13,14,15$

avoiding 38

manoeuvring envelopes 18, 19, 20

CSD 1 (circular deck/two-way-flow) 126, 127

static efficiency 127

cycle parking

lockers 164,165

stands for 165,166

decks

combined flat and sloping 91

directional markings 177

drainage falls 181

dynamic capacity $28-9$

exposed

effects of rain 26

roofing 33-34, 195-6

stopping distances on 27

temperature differences 196

waterproofing 33

flat, with internal ramps 103

frost prevention 198

gradients 25

level indicators 176,178 
decks (continued)

metal plate 196

surface abrasion 198

washing-down facilities 181

waterproofing 197-8

deflections, structural 198-9

design

aesthetics 200, 201-2, 202

angled stalls, implications 39-40, 39

briefs $4-5$

existing 37

questionnaires 4

design and build projects 201

Design recommendations for multi-storey and underground car parks (Des. Rec.) 1 ramps 16,16

dimensions

disabled parking stalls 162, 162

stalls

area per car space 31

length 9

width 9,27

disabled drivers

see also disabled pedestrians

flat and sloping decks 75

hillside car parks 161

separation of 163

sloping parking decks 59

tariffs for 163

disabled parking stalls 160

dimensions 162, 162

legal requirements 161

location

parameters $161-2$

random 162

numbers 161

supervision 162

disabled pedestrians

see also disabled drivers

fire escapes 156,183

lift buttons 177

ramps 15,156

refuges for 154

viewing panels 177

double helix

interlocking, one-way-flow 68, 69

one-way-flow

end connected 64,65

side connected $72,73,86,87$

two-way-flow, end connected 66,67

drainage

deck falls 181

gully outlets 198-9

location 181

petrol interceptors 181

protection of 180

roofs 181

stair cores 181

ventilating 181

washing-down 181

driver frustration

complex designs 37-8

potential conflict 49, 51, 129

stall searching 35,73

dry-risers 154,184

dynamic capacity

decks 28-9 entries 26

exits 26

ramps $27-8$

dynamic efficiency, angled stalls 29

efficiency see circulation efficiency; dynamic efficiency; static efficiency

emergency signs 179

lighting 175, 179, 183

entries

dynamic capacity 26

two-wheeled vehicles 165

environment, aesthetics 200, 201-2, 202

ER 1 (full circular ramps/two-way-flow) 140, 141 alternatives to 141

ER 2 (full circular ramps/one-way-flow) 142, 143 as alternative 141

alternatives to 143

ER 3 (straight ramps/one-way-flow) 144, 145 alternatives to 145

ER 4 (storey height straight ramps) 146, 147

ER 5 (stadium-shaped interlocking ramps) 148, 149

ER 6 (circular interlocking ramps) 150, 151

exit barriers, ticket insertion 38

exit routes

dynamic capacity 26

rapid 36

exits

emergency 183

ramps, headroom 15

two-wheeled vehicles 165

external ramps 138,139

see also ER series

FIR 1 (one-way-flow/two-way ramps/right angles) 102, 104, 105

alternatives to 105

circulation efficiency 35

static efficiency 105

FIR 2 (one-way-flow/scissors ramps) 106, 107

alternatives to 107

static efficiency 107

FIR 3 (one-way-flow/two-way ramps/parallel) 108, 109

alternatives to 109

static efficiency 109

FIR 4 (one-way-flow/one-way ramps) 110, 111

alternatives to 111

static efficiency 111

fire alarms 183

fire escapes

access to $153-4$

disabled pedestrians 156,183

distances from $155-6,183$

horizontal 155

routes to 183

stairs as $153,154-5$

fire fighting $182,183-4$

lifts 184

smoke containment 184

sprinklers 184

fire lobbies $154-5,154$

fire regulations, stairs as fire escapes 153

fire safety strategies 183

flat decks

external ramps, capacity 31

internal ramps 103 
flat decks with internal ramps see also FIR series multi-bin systems 103 ramp gradients 103

flat and sloping deck layouts 75

see also FSD series

disabled drivers 75

pedestrian movements 75

four-wheel drive (4WD) vehicles 6

Freyssinet, Eugene 1

frost prevention, decks 198

FSD 1 (single helix/two-way-flow) 76, 77

as alternative 79

alternatives to 77

static efficiency 77

FSD 2 (single helix/one-way-flow/rapid outflow) 78 , 79

as alternative 53

alternatives to 79

static efficiency 79

FSD 3 (combined helix/side connected/one- and two-way-flows) 80,81

as alternative 51,53

alternatives to 81

static efficiency 81

FSD 4 (combined helix/side connected/one-way-flow) 82,83

as alternative 51

alternatives to 83

static efficiency 83

FSD 5 (double helix/side connected/one-way-flow) 74 , 84,85

alternatives to 85

static efficiency 85

FSD 6 (double helix/side connected/one-way-flow) 86 , 87

FSD 7 (double helix/side connected/one-way-flow) 86 , 87

FSD 8 (single helix/one-way-flow/internal ramp) 88,89 alternatives to 89

static efficiency 89

gradients

parking decks 25,59

disabled drivers 59

sloping 59

ramps

pedestrian 24

vehicle $15,16-17,16,18,103$

single storey rise 103

ground clearances, standard design vehicles 7-8, 10

half external ramp types 128,129

see also HER series

capacity 31

driver conflict in 129

half external ramps, vehicle 126, 127

half spirals, one-way-flow types 130,131

headroom 25

light fittings 25

height

limitation gantries 25,25

standard design vehicles 6,10

helix see combined helix; double helix; single helix

HER 1 (half spiral/one-way-flow) 130, 131

HER 2 \& 3 (straight ramps/one-way-flow) 130, 131 alternatives to 133
HER 4 (straight ramps end located/one-way-flow) 134, 135

alternatives to 135

HER 5 (straight ramps end located/one-way-flow) 136, 137

alternatives to 137

hillside conditions

disabled drivers 161

multi-storey car parks 15

impacts

protection from 196-7

speeds 26,196

inhabited layouts, definition 33

interlocking double helix, one-way-flow type 68,69

interlocking ramps

circular type 24, 150, 151

stadium type 24, 148, 149

internal environmental monitoring 192

kerbs, pedestrian separation by $24-5$

lengths

parking stalls 9

standard design vehicles 6,10

levels, optimum numbers 33

lifts

buttons, disabled pedestrians 177

capacity per hour 158

considerations for 153

door widths 157,161

fire fighting 184

long stay car parks 153,157

medium stay car parks 153,157

short stay car parks 153,157

space requirements 157

supermarket requirements $156-7$

tidal car parks 157

lighting 174

controls 175

emergency $175,179,183$

signs 175,185

fittings, headroom 25

and painting 175

security 169,175

top decks 175

limosines

in multi-storey car parks 7

stretched 7

lockers

cycles 164,165

helmets/clothes 166

long stay car parks 9

capacities 31

flat and sloping decks 75

lifts 153,157

recommendations for $55,57,63,97$

main terminal car parks see long stay car parks

manoeuvring envelopes (ME)

historical $1-2,2$

ramps $18,19,20$

stall access 18, 19, 20

market values, multi-storey car parks 3

MD 1 (one-way-flow/circular end ramps) 112, 114, 115

as alternative 121

alternatives to 115 
MD 1 (one-way-flow/circular end ramps) (continued) static efficiency 115

variations 115

MD 2 (two-way-flow/one circular end ramps) 116, 117 alternatives to 117

static efficiency 117

variations 117

MD 3 (one-way-flow/10 stalls wide) 118, 119 static efficiency 119

MD 4 (two-way-flow/10 stalls wide) 118, 119 static efficiency 119

MD 5 (two-way-flow/sloping decks/10 stalls wide) 118, 119

static efficiency 119

MD 6-8 (one- and two-way-flows 8 stalls wide) 120, 121 as alternative 123

alternatives to 121

static efficiency 121

MD 9-11 (one- and two-way-flows/8 stalls wide/split levels) 122,123

alternatives to 123

static efficiency 123

medium stay car parks 9

capacities 31

large-capacity 45

lifts 153,157

recommendations for

combined flat and sloping decks 93, 97

sloping decks 61,73

split level decks 47,57

message signs, variable $34,67,81,89,115,178-9$

metal plate decks 196

minimum dimension layouts 113

see also MD series

underground 112, 113

motorcycle parking

free-standing 164, 165-6, 166

hard surface 166

helmet/clothes lockers 166

security surveillance 166

motorists' destinations, car park influences 1-2

multi-purpose vehicles (MPV) 6

multi-storey car parks (MSCP)

aisle viewing angles 8

categories 9

changes of use $2-3$

first 1

hillside conditions 15

market values 3

running costs 187

sale of 2-3

music, and security 169

natural ventilation 191

obstructions, between parking stalls 9-10

occupancy

maximum 154

notional 153-4

one- and two-way-flow types

combined, three + bins wide 52,53

combined helix

side connected 70, 71, 80, 81

eight stalls wide 120,121

split-level 122, 123

single ramp 98, 99

ten stalls wide 118,119 one-way-flow

aisle widths

minimum 13

reduced 11,19

circular ramps 22, 24

preference for 8

ramp widths $20-1$

one-way-flow types

see also one- and two-way-flow types

combined, three + bins wide 50,51

combined helix, side connected 82,83

contra-flow rapid exit 56,57

double helix

end connected 64,65

side connected $72,73,86,87$

end ramps 95,96

circular 114,115

excluded outflow 28-9, 29, 42, 46, 47

full circular ramps 142, 143

half spiral 130, 131

interlocking double helix 68, 69

internal ramps 92, 93

one-way ramps, separated 110,111

rapid outflow 44,45

capacity 31

circulation efficiency 35

scissors ramps 48,49

at right angles 106, 107

single helix

internal ramps 88,89

rapid outflow $63,64,78,79$

straight ramps 132, 133, 144, 145

end located 134, 135, 136, 137

two-way ramps

at right angles 104, 105

parallel 108, 109

warped decks 100, 101

outflow

excluded rapid 46,47

rapid $44,45,62,63$

overhead signage 176,177

painting, light colours 175

parking stalls see stalls

partially sighted, guidelines 177

pay and display 187

pay stations, signs 178

payment

by mobile phone $187-8$

on exit 186,187

on foot 187

pedestrians

see also disabled pedestrians

access flat and sloping decks 91

angled stalls 11

encumbered 26

fire escapes

access to $153-4,183$

distances from 155-6, 183

stairs as $153,154-5$

flat and sloping decks 75

guard rails 197

lifts 153

ramps 16

gradients 24

layouts 156, 156

split-level decks 43 
signage 177-8

sloping decks 59

and vehicle ramps 15

kerb separation 24-5

petrol interceptors, drainage 181

plans, availability 178

powered two wheelers (PTW)

facilities for 164,165

separate entries and exits 165

rain

effects on decks 26

effects on stopping distances 27

ramps (pedestrian) 16

gradients 24

regulations 43

split-level decks 43

ramps (vehicle)

see also access ways

aisles, projections into 17

circular 22, 24

one-way-flow 142, 143

two-way-flow 140, 141

cross- $13,14,15$

dynamic capacities $27-8$

end 95,97

circular 114, 115, 116, 117

exits, headroom 15

gradients 15

recommended 16-17, 16, 18

ground clearance on 7-8

half external 126,127

interlocking

circular type 24, 150, 151

stadium type $24,148,149$

internal 88,89

internal cross- 91

manoeuvring envelopes 18, 19, 20

open-aspect 14

outer clearances 14,15

pedestrians and vehicle 15

scissor-type 22, 23

scissors, one-way-flow types 48, 49, 106, 107

separated, one-way-flow types 110,111

side-by-side 22

storey height $17,18,103,105$

straight

one-way-flow 132, 133, 134, 135, 136, 137, 144, 145

storey height 146,147

two-way, one-way-flow types 104, 105, 108, 109

widths

and aisle entry efficiency 21

angled stalls 21

one-way-flow 19, 20-1

turning circles 22,23

two-way-flow 22

rapid exit routes $36,78,79$

refuges, disabled pedestrians 154

reinforced concrete structures 195

finishes 197

life expectation 195

shrinkage joints 196

retail outlets

short stay car parks 26

supermarkets, lift requirements 156-7 roofs

exposed decks 33-4, 195-6

drainage 181

running costs, multi-storey car parks 187

Safer Car Parks scheme 169

sales, multi-storey car parks 2-3

scissor-type ramps 22, 23

one-way-flow (SLD 3) 48, 49

scissors ramps

one-way-flow types 48,49

at right angles 106, 107

SD 1 (single helix/two-way-flow) 60, 61

as alternative $55,63,77,97$

alternatives to 61

congestion 61

static efficiency 61

SD 2 (single helix/one-way-flow/rapid outflow) 62, 63

alternatives to 63

static efficiency 63

SD 3 (double helix/end connected/one-way-flow) 64, 65

as alternative $67,69,89,101$

alternatives to 65

static efficiency 65

SD 4 (double helix/end connected/two-way-flow) 66, 67

as alternative $67,69,89$

alternatives to 67

static efficiency 67

SD 5 (interlocking double helix/one-way-flow) 58, 68, 69 as alternative $51,53,67,89$

static efficiency 69

SD 6 (combined helix/side connected/one- and two-way-flows) 70,71

alternatives to 71

static efficiency 71

SD 7 (double helix/side connected/one-way-flows) 72 , 73

alternatives to 73

static efficiency 73

SD 8 (double helix/side connected/one-way-flows) 72,73

alternatives to 73

static efficiency 73

searching

stalls

inefficient 51, 53, 81, 83

traffic congestion 73,81

security

CCTV 168, 169-70

car park shapes 170

optimum monitoring $169-70$

presence of 169

lighting 169

motorcycle parking 166

music as aid 169

public perceptions $169,170,175$

women-only car parks 170

short stay car parks 9

capacities 31

large-capacity 45

lift requirements $156-7$

lifts 153,157

recommendations for

combined flat and sloping decks 93, 97

sloping decks 61,73

split-level decks 47,57

retail outlets 26

side-by-side ramps 22 
signage

control 178

deck levels, indications 176,178

deck markings, directional 177

emergency 179

lighting 175

headroom 25

overhead 176, 177

pay stations 178

pedestrians $177-8$

schedule 179

variable message $34,67,81,89,115,178-9$

single helix

one-way-flow

internal ramps 88,89

rapid outflow $62,63,78,79$

two-way-flow

flat and sloping decks 76, 77

sloping decks 60,61

SLD 1 (one-way-flow/rapid outflow) 44, 45

as alternative 47,57

alternatives to 45

capacity 31,45

circulation efficiency 35,45

static efficiency 45

SLD 2 (one-way-flow/excluded outflow)

as alternative

to FSD series 89

to $\mathrm{SD}$ series $65,67,71,73$

to SLD series 45

to VCM series 95

alternatives to 47

static efficiency 47

SLD 3 (one-way-flow/scissors ramps) 48, 49

as alternative $81,85,93$

alternatives to 49

capacity 31

static efficiency 49

SLD 4 (combined one-way-flows, three + stalls wide) 50,51

alternatives to 51

circulation efficiency 51

SLD 5 (combined one- and two-way-flows, $3+$ bins wide) 52,53

as alternative 83

alternatives to 53

static efficiency 53

SLD 6 (two-way-flow/combined ramps) 54, 55

as alternative $49,61,77$

alternatives to 55

static efficiency 55

SLD 7 (one-way-flow/contra-flow exit) 56, 57

as alternative 97,99

alternatives to 57

static efficiency 57

sloping parking decks (SD)

see also SD series

definition 59

disabled drivers 59

parking gradients 59

pedestrian considerations 59

smoke

control 184, 192

detectors 192

speed limits, imposition of 27

split-level decks (SLD)

see also SLD type series advantages 43

pedestrian ramps in 43

popularity 43

sports utility vehicles (SUV) 6

sprinklers 184

staff parking see tidal car parks

stairs

cores, drainage 181

as fire escapes

fire lobbies $154-5,154$

fire regulations 153

widths 155

stalls

see also angled stalls; disabled parking stalls; parking decks

access, manoeuvring envelopes 18, 19, 20

dimensions

area per car space 31

length 9

width 9,27

driver searches 35

dynamic capacity 27

obstructions between 9-10

rectangle 6,9

searching

inefficient 51, 53, 81, 83

traffic congestion 73,81

static efficiency $30-1$

standard design vehicles (SDV)

see also vehicles

95 factor 6,10

departures from 6,7

ground clearance 7-8, 10

height 6,10

length 6,10

turning diameters 8,10

wheelbase 7,10

width 6,10

static efficiency

definition 30

external bins 30

internal bins 31

single bins 30

two-bin layout 30

steelwork 195

coatings 197

storey height ramps 17, 18, 103, 105

structure

alternative materials 195

deflections 198-9

reinforced concrete 195

finishes 197

life expectation 195

shrinkage joints 196

steelwork 195

coatings 197

supermarkets, lift requirements 156-7

surveillance see CCTV; lighting; security

swept paths, turning circles 22,23

tag systems of payment 188

tariffs see control systems

temperature differences, exposed decks 196

tidal car parks 9, 49

capacities 32

with flow reversal 69

lifts 157 
recommendations for

flat and sloping decks 97

sloping decks $61,63,65,69$

split level decks 49, 55, 57

two-way-flow, ramps 21-2

top decks see decks, exposed

turning circles

circular ramp systems 139

minimum dimension layouts 113

standard design vehicles 8,10

swept paths 22,23

two-bin layout, static efficiency 30

two-way-flow

aisle widths

minimum 13

reduced 12

circular decks 126, 127

circular ramps 24

end 116,117

full $140,141,142,143$

traffic congestion 61

vehicles crossing 26-7

two-way-flow types

see also one-and two-way-flow types

with combined ramps 54,55

double helix, end connected 66,67

single end ramp 96, 97

single helix, sloping decks 60,61

underground parking 172

constraints 173

efficiency 173

minimum dimension layouts 112, 113

ventilation $190,191-2$

uninhabited layouts, definition 33

USA, high level parking 33, 34

user-friendly car parks 2, 37-9

user-unfriendly car parks 125

variable message signs $34,67,81,89,115,178-9$

VCM 1 (one-way-flow/internal ramps) 90, 92, 93 as alternative

to FIR series $105,107,109,111$

to FSD series $81,83,85,89$

to $\mathrm{SD}$ series $65,67,71,73$

to SLD series $45,47,51,53,57$

to WPD series 101

within VCM series 95, 99

alternatives to 93 capacity 31

static efficiency 93

VCM 2 (one-way-flow/end ramps) 94, 95

as alternative

to FSD series 85,89

to $\mathrm{SD}$ series $65,67,71,73$

to SLD series 57

within VCM series 93, 99

alternatives to 95

static efficiency 95

VCM 3 (two-way-flow/single ramp) 96, 97

as alternative $49,55,61,77$

alternatives to 97

static efficiency 97

VCM 4 (one- and two-way-flow/single ramp) 98, 99

as alternative 57,61

alternatives to 99

static efficiency 99

vehicles

see also standard design vehicles

camper vans 6

four-wheel drive 6

limosines

in multi-storey car parks 7

stretched 7

new registrations by type 203

sports utility 6

ventilation

air change rates 192

fans $190,191-2$

natural 191

underground parking 190, 191-2

viewing panels, for disabled pedestrians 177

warped parking decks 91

see also WPD series

washing-down facilities, decks 181

waterproofing decks 197-8

wheelbase, standard design vehicles 7,10

widths

aisles $10-11$

minimum 13

parking stalls 9

stairs 155

standard design vehicles 6,10

women-only car parks 170

WPD 1 (warped deck/one-way-flow) 100, 101

alternatives to 101

static efficiency 101 